\title{
Studies on blends of binary crystalline polymers: Miscibility and crystallization behavior in PBT/PAr(I27-T73)
}

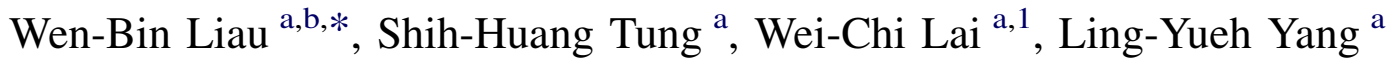 \\ ${ }^{a}$ Department of Materials Science and Engineering, National Taiwan University, No. 1, Roosevelt Road, Sec. 4, Taipei, Taiwan 10617, ROC \\ ${ }^{\mathrm{b}}$ Institute of Polymer Science and Engineering, National Taiwan University, No. 1, Roosevelt Road, Sec. 4, Taipei, Taiwan 10617, ROC
}

Received 27 June 2006; received in revised form 4 September 2006; accepted 24 September 2006

Available online 20 October 2006

\begin{abstract}
The miscibility and crystallization behavior of binary crystalline blends of poly(butylene terephthalate) [PBT] and polyarylate based on Bisphenol A and a 27/73 mole ratio of isophthalic and terephthalic acids [PAr(I27-T73)] have been investigated by differential scanning calorimetry (DSC). This blend system exhibits a single composition-dependent glass transition temperature over the entire composition range. The equilibrium melting point depression of PBT was observed, and Flory interaction parameter $\chi_{12}=-0.96$ was obtained. These indicate that the blends are thermodynamically miscible in the melt. The crystallization rate of PBT decreased as the amount of PAr(I27-T73) increased, and a contrary trend was found when PAr(I27-T73) crystallized with the increase of the amount of PBT. The addition of high- $T_{\mathrm{g}}$ PAr(I27-T73) would suppress the segmental mobility of PBT, while low- $T_{\mathrm{g}}$ PBT would have promotional effect on PAr(I27-T73). The crystallization rate and melting point of PBT were significantly influenced when the PAr(I27-T73) crystallites are previously formed. It is because not only does the amorphous phase composition shift to a richer PBT content after the crystallization of PAr(I27-T73), but also the PAr(I27-T73) crystal phase would constrain the crystallization of PBT. Thus, effects of the glass transition temperature, interaction between components, and previously formed crystallites of one component on the crystallization behavior of the other component were discussed and compared with blends of PBT and PAr(I-100) based on Bisphenol A and isophthalic acid.

(C) 2006 Elsevier Ltd. All rights reserved.
\end{abstract}

Keywords: Crystallization; Crystalline/crystalline blends; Miscibility

\section{Introduction}

Binary polymer blends can be classified into amorphous/ amorphous, crystalline/amorphous, and crystalline/crystalline systems based on the crystallizability of the constituents. Most of the previous research focused on the polymeric mixture containing two amorphous components [1-3]. Polymer blends

\footnotetext{
* Corresponding author. Department of Materials Science and Engineering, National Taiwan University, No. 1, Roosevelt Road, Sec. 4, Taipei, Taiwan 10617, ROC. Tel.: +88622362 6119; fax: +8862 23634562 .

E-mail address: wbliau@ccms.ntu.edu.tw (W.-B. Liau).

${ }^{1}$ Present address: Department of Chemical and Materials Engineering, Tamkang University, No. 151, Ying-chuan Road, Tamsui, Taipei County, Taiwan 25137, ROC
}

containing two crystalline components were less frequently discussed.

Polyarylates based on Bisphenol A and isophthalic acid [PAr(I-100)] are not commercially available now. However, its homologous copolymers, polyarylates based on Bisphenol A and isophthalic and terephthalic acids, have been commercialized and recognized as important materials with high distortion temperature, excellent mechanical properties, and toughness, especially the ultraviolet (UV) resistance after long-time exposure [4-6]. The polymer blends of PBT and commercialized polyarylates have been studied intensively [7-9]. However, most of these studies focused on the blends of crystalline PBT and amorphous polyarylates. Recently, the PBT/PAr(I-100) blends containing two crystalline components have been investigated and documented in the literature. Liu et al. $[10,11]$ reported the miscibility and crystallization 
behavior of PBT and PAr(I-100) blends, a crystalline/crystalline blend system, which have been investigated by differential scanning calorimetry (DSC). Judging from the melting point depression and single glass transition temperature of the blends, this system is miscible over the whole composition range in the melt. The crystallization rate of $\mathrm{PAr}(\mathrm{I}-100)$ is much faster when the PBT was added. The crystallization of PAr(I-100) is significantly influenced when the PBT crystallites are previously formed. On the other hand, the crystallization rate of PBT is reduced with the addition of PAr(I-100). In this study, the blends are poly(butylene terephthalate) [PBT] and polyarylate [PAr(I27-T73)], a semicrystalline copolymer of Bisphenol A and a 27/73 mole ratio of isophthalic and terephthalic acids. Although both PAr(I-100) and PAr(I27-T73) are semicrystalline polymers, the glass transition temperature of PAr(I27-T73) is about $217^{\circ} \mathrm{C}$, while the glass transition temperature of $\operatorname{PAr}(\mathrm{I}-100)$ is about $180^{\circ} \mathrm{C}$. Furthermore, the interaction between PAr(I27-T73) and PBT is different from that between PAr(I-100) and PBT. The purpose of the present study is to understand the nature of miscibility and crystallization behavior in the crystalline/crystalline polymer blends. Also, the effects of glass transition temperature, the interaction between components, and the previously formed crystallites of one component on the crystallization behavior of the other component are studied by comparing PBT/PAr(I-100) and PBT/PAr(I27T73). To prevent the degradation of PBT during the heating, PAr(T-100) based on Bisphenol A and terephthalic acid was not chosen to study due to the high melting temperature.

\section{Experimental section}

\subsection{Materials and preparing method of samples}

The PBT sample was obtained from Nan Ya Plastics Co. Inc., and its number-average molecular weight was 27,400. The PAr(I27-T73) used in this study was synthesized by feeding Bisphenol A with isophthaloyl chloride and terephthaloyl chloride via interfacial polymerization. The detailed preparation and reaction mechanism were demonstrated in Chu and Lee's [12] work. The backbone structure of PAr(I27-T73) was determined by NMR. The ratio of isophthalic/terephthalic segments was $27 / 73$.

Blends of PBT and PAr(I27-T73) were prepared by dissolving PBT and PAr(I27-T73) in $m$-cresol. The blends were subsequently recovered by precipitating them in a 10-fold excess volume of methanol. The blends were then washed with a large amount of methanol. To remove the residual solvent, the blends were dried in vacuum at $200{ }^{\circ} \mathrm{C}$ for $24 \mathrm{~h}$.

\subsection{Measurements}

Thermal transitions of PBT/PAr(I27-T73) blends were measured with a TA Instruments DSC 2010 differential scanning calorimeter equipped with a liquid nitrogen cooling system. Indium was used as the standard for temperature calibration. Samples of 5-10 mg were used in all experiments. To erase the previous thermal history, the samples were heated above the melting point $\left(330{ }^{\circ} \mathrm{C}\right)$. For the measurement of glass transition, the samples were quenched into liquid nitrogen. At a heating rate of $20{ }^{\circ} \mathrm{C} / \mathrm{min}$, the melt-quenched samples were heated from $0{ }^{\circ} \mathrm{C}$ to $330{ }^{\circ} \mathrm{C}$, and the glass transition temperature and enthalpy of recrystallization and melting would be observed. The glass transition temperature was determined from the half-height point of the step change in the thermogram, and enthalpy of recrystallization and melting was obtained from the second run.

The isothermal crystallization of PBT/PAr(I27-T73) blends was also measured by the TA DSC 2010. The samples were heated to $330{ }^{\circ} \mathrm{C}$ and held for $1 \mathrm{~min}$ on a Linkam THMS600 hot stage and then quickly moved into the DSC cell, where the temperature was kept at $250{ }^{\circ} \mathrm{C}$. The isothermal crystallization was recorded till the crystallization peak was complete.

X-ray diffraction patterns were recorded by a Philips PW-1710 diffractometer with Ni-filtered $\mathrm{Cu} \mathrm{K} \alpha$ radiation. The samples were prepared by compression molding.

\section{Results and discussion}

\subsection{Miscibility of PBT/PAr(I27-T73) blends}

Before investigating thermal properties of PBT/PAr(I27T73) blends, the transesterification reaction must be discussed briefly. It is well known that transesterification in polyester blends can affect the thermal properties, such as melting point, crystallization and so on $[13,14]$. To examine the transesterification effect on the crystallization behavior of the samples, the PBT/PAr(I27-T73) blend with 85/15 weight ratio was chosen for testing. The sample was heated to $330^{\circ} \mathrm{C}$ and held for different time. Then, the sample was quenched to $150{ }^{\circ} \mathrm{C}$ for the isothermal crystallization of PBT for $24 \mathrm{~h}$. In such condition, PAr(I27-T73) crystallites did not form. The melting peak and endotherm peak of the sample were examined utilizing DSC. The PBT melting point and endotherm peak of $85 / 15$ blend did not change when it was held at $330{ }^{\circ} \mathrm{C}$ for $1 \mathrm{~min}$. However, samples heated to $330{ }^{\circ} \mathrm{C}$ and held for $3 \mathrm{~min}$ exhibited the slight depression of melting point and melting enthalpy of PBT. This series of experiments confirm that samples held at $330{ }^{\circ} \mathrm{C}$ for $1 \mathrm{~min}$ would not be influenced by transesterification. X-ray diffraction patterns showed that PBT and PAr(I27-T73) could completely melt when the samples were heated to $330{ }^{\circ} \mathrm{C}$ and held for $1 \mathrm{~min}$. Therefore, in this study all samples were heated to $330{ }^{\circ} \mathrm{C}$ for $1 \mathrm{~min}$ to eliminate thermal history.

DSC thermograms of PBT/PAr(I27-T73) blends are shown in Fig. 1. Samples were quenched from $330{ }^{\circ} \mathrm{C}$ into liquid nitrogen, and then scanned to $330{ }^{\circ} \mathrm{C}$ by the rate of $20^{\circ} \mathrm{C} / \mathrm{min}$. The glass transition temperatures of melt-quenched $\mathrm{PBT} /$ PAr(I27-T73) blends observed in DSC scans are indicated by arrows. The glass transition temperature of neat PBT is $43.67{ }^{\circ} \mathrm{C}$. Compared with other reports, it is reasonable. The glass transition temperature of PBT between $30{ }^{\circ} \mathrm{C}$ and $45{ }^{\circ} \mathrm{C}$ has been reported from DSC measurements [7,14], which is independent of crystallinity, as described by Illers [15]. 


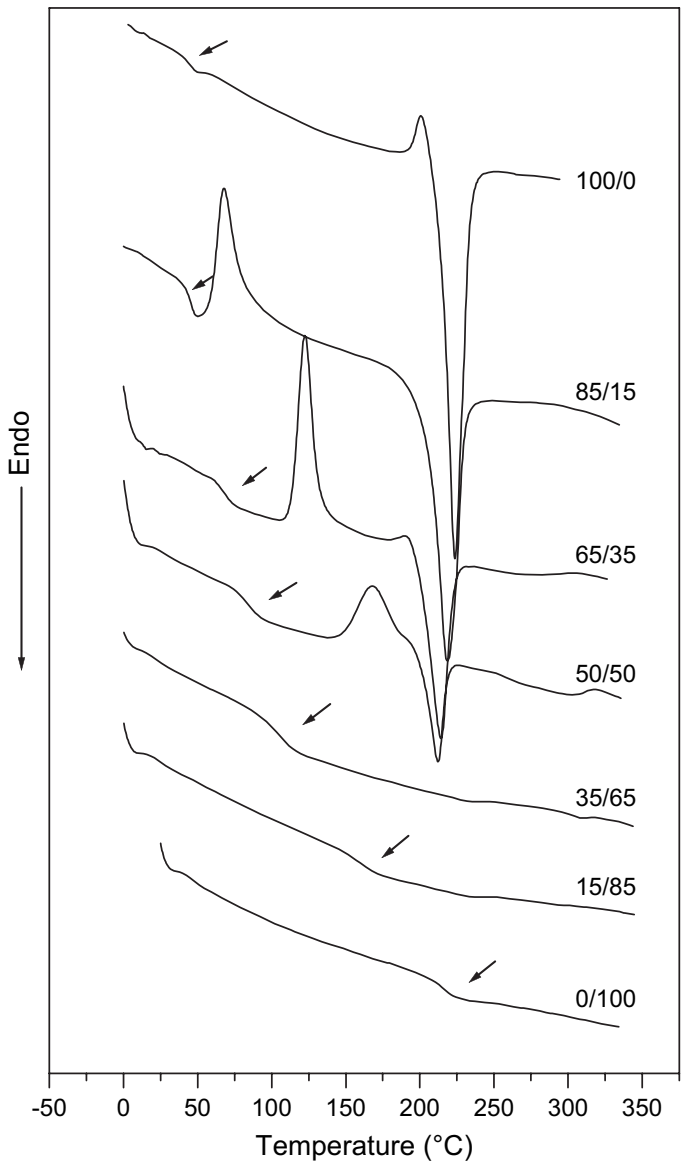

Fig. 1. The DSC traces $\left(20^{\circ} \mathrm{C} / \mathrm{min}\right)$ from second run of PBT/PAr(I27-T73) blends; $T_{\mathrm{g}}$ is indicated by an arrow.

The glass transition temperature of neat $\operatorname{PAr}(\mathrm{I} 27-\mathrm{T} 73)$ is $217.18^{\circ} \mathrm{C}$, which is greater than that of neat $\operatorname{PAr}(\mathrm{I}-100)$, $180.39^{\circ} \mathrm{C}$. Fig. 1 shows that a single composition-dependent $T_{\mathrm{g}}$ with intermediate value between their respective neat states was found for each blend, and this indicates that PBT and PAr(I27-T73) are miscible in the melt. Certainly, glass transition temperatures of PBT/PAr(I27-T73) are greater than those of PBT/PAr(I-100) at the same composition. Heats of recrystallization and melting of PBT were calculated and are listed

Table 1

DSC scanning data of PBT/PAr(I27-T73) blends

\begin{tabular}{llllll}
\hline $\begin{array}{l}\text { PBT/PAr(I27-T73) } \\
\text { (wt\%/wt\%) }\end{array}$ & $\begin{array}{l}\text { Amorphous } \\
T_{\mathrm{g}}\left({ }^{\circ} \mathrm{C}\right)\end{array}$ & \begin{tabular}{l} 
PBT phase \\
\cline { 3 - 6 }
\end{tabular} & & & \multicolumn{3}{c}{$\Delta H_{\mathrm{c}} / \Delta H_{\mathrm{m}}{ }^{\mathrm{a}}$} & $\Delta H_{\mathrm{c}}-\Delta H_{\mathrm{m}}{ }^{\mathrm{b}}$ & $\begin{array}{l}\Delta H_{\mathrm{m}}{ }^{\prime} \\
\mathrm{wt}^{\mathrm{c}}{ }^{\mathrm{c}}\end{array}$ & $\begin{array}{l}T_{\mathrm{m}} \\
\left({ }^{\circ} \mathrm{C}\right)\end{array}$ \\
\hline $100 / 0$ & 43.67 & 0.05 & 53.60 & 56.72 & 223.51 \\
$85 / 15$ & 45.05 & 0.52 & 26.39 & 65.31 & 218.36 \\
$65 / 35$ & 68.37 & 0.93 & 2.09 & 46.30 & 214.07 \\
$50 / 50$ & 84.54 & $1.00^{\mathrm{d}}$ & $0.00^{\mathrm{d}}$ & $35.34^{\mathrm{d}}$ & 212.33 \\
$35 / 65$ & 106.47 & n.a. & n.a. & n.a. & n.a. \\
$15 / 85$ & 159.21 & n.a. & n.a. & n.a. & n.a. \\
$0 / 100$ & 217.18 & n.a. & n.a. & n.a. & n.a. \\
\hline
\end{tabular}

${ }^{a}$ Ratio of exothermic and endothermic heats (dimensionless).

${ }^{\mathrm{b}}$ Difference of endothermic and exothermic heats $(\mathrm{J} / \mathrm{g})$.

${ }^{c}$ Endothermic heats of melt corrected by PBT weight fractions $(\mathrm{J} / \mathrm{g})$.

${ }^{\mathrm{d}}$ Calculated from curve fitting.

e Not available. in Table 1. Note that heats of recrystallization and melting of PBT/PAr(I27-T73) 50/50 were calculated from the result of curve fitting due to the overlap of a recrystallization peak of $\operatorname{PAr}($ I27-T73) and the recrystallization and melting peaks of PBT. Because PAr(I27-T73) crystallized very slowly, the endothermic peak observed around $300{ }^{\circ} \mathrm{C}$ in $50 / 50$ appeared to be the melting of PAr crystals formed during heating instead of quenching. Thus, the enthalpies of recrystallization and melting of PAr(I27-T73) were adjusted to be the same. The same assumption was also used for PBT. Since the crystallization of PBT in 65/35 during quenching was already slight $\left(\Delta H_{\mathrm{c}} l\right.$ $\left.\Delta H_{\mathrm{m}}=0.93\right)$, it seemed reasonable that PBT in 50/50 could crystallize only during heating as the weight fraction of PAr(I27-T73) was further increased from 35\% to 50\%. Fitting with the above conditions yielded the best result. Attempts were made as well to fit the DSC trace without the PAr recrystallization but all failed. Fig. 2 shows the result of curve fitting of PBT/PAr(I27-T73) 50/50 from DSC trace of Fig. 1 between $130{ }^{\circ} \mathrm{C}$ and $230{ }^{\circ} \mathrm{C}$.

The dependence of $T_{\mathrm{g}}$ on blend composition can be estimated using classical Fox's law [16] (Eq. (1)), or GordonTaylor's equation [17] (Eq. (2)):

$\frac{1}{T_{\mathrm{g}, \text { blend }}}=\frac{w_{1}}{T_{\mathrm{g}, 1}}+\frac{w_{2}}{T_{\mathrm{g}, 2}}$

$T_{\mathrm{g}, \text { blend }}=\frac{w_{1} T_{\mathrm{g}, 1}+k w_{2} T_{\mathrm{g}, 2}}{w_{1}+k w_{2}}$

where $w_{i}$ is the weight fraction, $k$ is the fitting parameter that equals $\Delta \alpha_{2} / \Delta \alpha_{1}\left(\Delta \alpha_{i}\right.$ is the difference between the volume expansion coefficient in the rubbery and glassy states), $T_{\mathrm{g}, i}$ is the glass transition temperature of the neat component, and subscripts 1 and 2 denote the PBT and PAr(I27-T73), respectively.

Fig. 1 and Table 1 show that crystallization of PBT in some blends was inevitable during the quenching. Thus, the amorphous composition must be corrected to utilize Fox's law or Gordon-Taylor's equation. The crystallinity of PBT in meltquenched samples was calculated from the following equation:

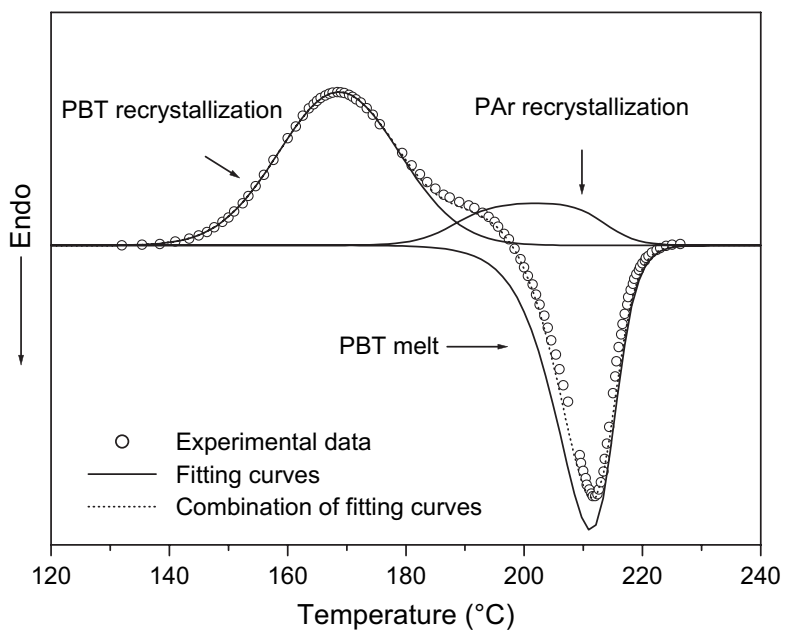

Fig. 2. Curve fitting of PBT/PAr(I27-T73) 50/50 from DSC trace of Fig. 1 between $130{ }^{\circ} \mathrm{C}$ and $230{ }^{\circ} \mathrm{C}$. 
$x_{\mathrm{c}, \mathrm{PBT}}=\frac{\Delta H_{\mathrm{m}, \mathrm{PBT}}-\Delta H_{\mathrm{c}, \mathrm{PBT}}}{\Delta H_{\mathrm{f}, \mathrm{PBT}}^{\mathrm{o}}}$

where $\Delta H_{\mathrm{c}, \mathrm{PBT}}$ is the recrystallization exotherm, $\Delta H_{\mathrm{m}, \mathrm{PBT}}$ is the melting endotherm, and $\Delta H_{\mathrm{f}, \mathrm{PBT}}^{\mathrm{o}}$ is the fusion heat of $100 \%$ crystalline PBT $(142 \mathrm{~J} / \mathrm{g})$ [15]. Then, the composition of amorphous phase was corrected by removing these previously formed PBT crystalline fractions. The crystallinity of PAr(I27-T73) in the melt-quenched sample was not able to be calculated due to the lack of the heat of fusion of $100 \%$ crystalline PAr(I27-T73). However, the crystallization of PAr(I27-T73) during quenching was very slight. Thus, the correction of amorphous composition due to the crystallization of PAr(I27-T73) during the quenching was neglected.

The predictions of Fox's law (solid line) and GordonTaylor's equation (dash line) are shown in Fig. 3. It is obvious that the $T_{\mathrm{g}}$-composition variation does not conform to Fox's equation but can be well described by Gordon-Taylor's equation with $k=0.3$.

The miscibility of polymer blends is usually determined by the observation of a single glass transition temperature. Furthermore, for blends containing a crystalline polymer, the melting point depression is also an indication of a miscible system. The equilibrium melting point was determined by HoffmanWeeks analysis. The equation is written in the following form:

$T_{\mathrm{m}}=\frac{1}{\gamma} T_{\mathrm{c}}+\left(1-\frac{1}{\gamma}\right) T_{\mathrm{m}}^{\mathrm{o}}$

where $T_{\mathrm{m}}$ and $T_{\mathrm{m}}^{\mathrm{o}}$ are the experimental melting temperature and equilibrium melting temperature of PBT in the blend, respectively, $T_{\mathrm{c}}$ is the crystallization temperature, and $\gamma$ is the proportional factor between the initial thickness of a chainfolded lamella, $l_{\mathrm{g}}{ }^{*}$, and final thickness, $l_{\mathrm{c}}$. At the beginning of the crystallization, the lamella thickness is $l_{\mathrm{g}}{ }^{*}$. As the crystallization proceeds, the lamella thickens, and the thickness becomes $l_{\mathrm{c}}$ at the end of the crystallization. Thus, $\gamma$ is also known as the thickening ratio.

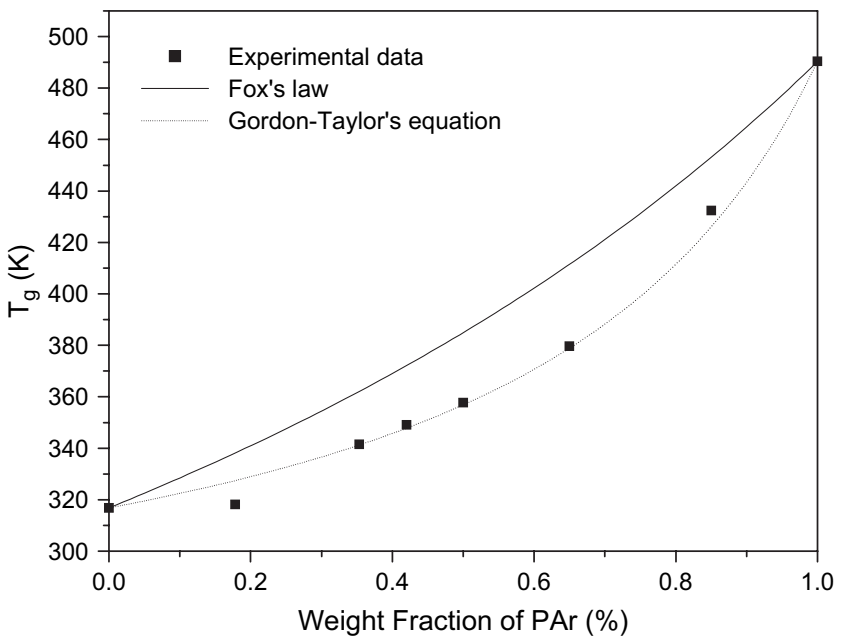

Fig. 3. Glass transition temperature versus composition of PBT/PAr(I27-T73) blends; $k=0.3$ in Gordon-Taylor's equation.

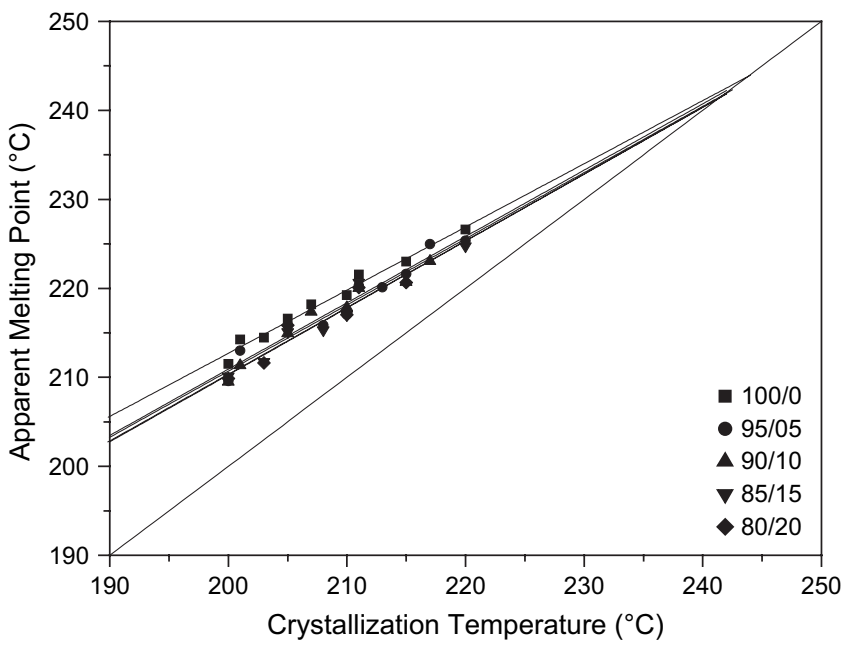

Fig. 4. Hoffman-Weeks plots of PBT/PAr(I27-T73) blends.

The equilibrium melting point, $T_{\mathrm{m}}^{\mathrm{o}}$, was obtained from the extrapolation of the $T_{\mathrm{m}}-T_{\mathrm{c}}$ plot to the $T_{\mathrm{m}}=T_{\mathrm{c}}$ line. Fig. 4 shows the Hoffman-Weeks plots of PBT/PAr(I27-T73) blends, where experimental data were obtained by isothermal crystallization for $12 \mathrm{~h}$. However, PAr(I27-T73) in the blends could become crystalline after long-term annealing if its weight ratio is greater than 0.35. To prevent PAr(I27-T73) crystallites from affecting PBT melting point, only PBT/ $\operatorname{PAr}(\mathrm{I} 27-\mathrm{T} 73)=100 / 0,95 / 05,85 / 15$, and 80/20 were used. The values of $T_{\mathrm{m}}^{\mathrm{o}}$ and $\gamma$ are listed in Table 2. It was found that the equilibrium melting point of PBT decreased with increasing PAr(I27-T73) content. The equilibrium melting point of PBT is reasonable in comparison with the data reported by Kimura [14] $\left(240^{\circ} \mathrm{C}\right)$, Cheng [18] $\left(245^{\circ} \mathrm{C}\right)$, and Liu [10] $\left(241^{\circ} \mathrm{C}\right)$ but is lower than that reported by Cebe and Huo [9] $\left(249^{\circ} \mathrm{C}\right)$. From Table 2 one can find that the thickening ratio, $\gamma$, decreases as PAr(I27-T73) content increases. This indicates that the PBT crystals become less stable because of the smaller lamellar thickness.

The melting point depression of a crystalline phase with noncrystalline polymeric diluent in a miscible blend was derived by Nishi and Wang [19]. The equation can be written as

$\frac{1}{T_{\mathrm{m}}^{\mathrm{o}}}-\frac{1}{T_{\mathrm{m}}^{\mathrm{o} \prime}}=\frac{-R V_{2}}{\Delta H_{\mathrm{f}}^{\mathrm{o}} V_{1}}\left[\frac{\ln \phi_{2}}{M_{2}}+\left(\frac{1}{M_{2}}-\frac{1}{M_{1}}\right) \phi_{1}\right]-\frac{R V_{2}}{\Delta H_{\mathrm{f}}^{o} V_{1}}\left(\chi_{12} \phi_{1}^{2}\right)$

where $V$ is the molar volume of the polymer repeating unit, $\phi$ is the volume fraction of the component in the blend, $\Delta H_{\mathrm{f}}^{\mathrm{o}}$ is

Table 2

Equilibrium melting point and $\gamma$ values of PBT for PBT/PAr(I27-T73) blends

\begin{tabular}{lll}
\hline PBT/PAr(I27-T73) & $\begin{array}{l}\text { Equilibrium melting } \\
\text { point } T_{\mathrm{m}}^{\mathrm{o}}\left({ }^{\circ} \mathrm{C}\right)\end{array}$ & $\gamma$ \\
\hline $100 / 0$ & 243.74 & 1.41 \\
$95 / 05$ & 242.93 & 1.34 \\
$90 / 10$ & 241.73 & 1.34 \\
$85 / 15$ & 241.37 & 1.33 \\
$80 / 20$ & 241.30 & 1.33 \\
\hline
\end{tabular}


the perfect crystal heat of fusion of the crystallizable polymer, $M$ is the degree of polymerization, $R$ is the universal gas constant, $T_{\mathrm{m}}^{\mathrm{o}}$ is the equilibrium melting point of pure crystalline polymer, $T_{\mathrm{m}}^{\mathrm{o} /}$ is the equilibrium melting point of a blend, and $\chi_{12}$ is the polymer/polymer interaction parameter. Subscripts 1 and 2 denote the amorphous and crystalline components, respectively. If the molecular weights of both components of blends are large enough, the entropy of mixing can be negligible; the melting point depression is dominated by the enthalpic term, and then the equation reduces to

$\frac{1}{T_{\mathrm{m}}^{\mathrm{o}}}-\frac{1}{T_{\mathrm{m}}^{\prime \prime}}=-\frac{R V_{2}}{\Delta H_{\mathrm{f}}^{\mathrm{o}} V_{1}}\left(\chi_{12} \phi_{1}^{2}\right)$

It is well known that experimental factors such as scanning rate, crystallization temperature range, and time of crystallization would affect the values obtained. Fig. 5 was plotted to obtain $\chi_{12}$ from Eq. (6). The following parameters were used: $\Delta H_{\mathrm{f}}^{\mathrm{o}}=31.2 \mathrm{~kJ} / \mathrm{mol}$-monomer [15], $V_{1}=266.2 \mathrm{~cm}^{3} / \mathrm{mol}$ monomer, and $V_{2}=129.6 \mathrm{~cm}^{3} / \mathrm{mol}-$ monomer. The molar volume of $\operatorname{PAr}(\mathrm{I} 27-\mathrm{T} 73)$ was assumed the same as that used by Kimura and Porter [14]. From the slope of the curve, it was found that $\chi_{12}=-0.96$. Because the value of $\chi_{12}$ is negative, it reconfirms that the polymeric mixture is thermodynamically miscible in the melt. The interaction parameter of PBT/PAr(I-100) was reported [10] as $\chi_{12}=-1.3$. Obviously, the interaction between PBT and PAr(I27-T73) is weaker than that between PBT and $\operatorname{PAr}(\mathrm{I}-100)$. The degree of miscibility is higher for PBT and PAr(I-100). Furthermore, Table 3 shows that the melting point depressions of PBT are greater for PBT/PAr(I-100) with the same composition.

\subsection{Crystallization and melting behavior of PBT/ PAr(I27-T73) blends}

In Fig. 1, PBT/PAr(I27-T73) blends with compositions of $100 / 0$ and $85 / 15$ both show one endothermic peak at around

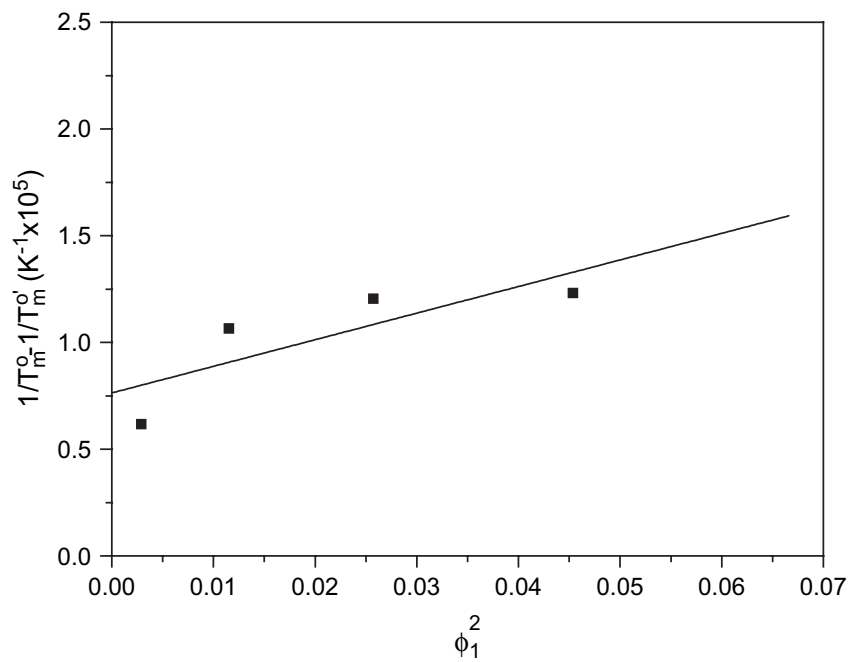

Fig. 5. Relative plot of melting point depression and volume fraction according to Nishi-Wang equation.
Table 3

Equilibrium melting point depression of PBT for PBT/PAr(I27-T73) and PBT/ PAr(I-100)

\begin{tabular}{lll}
\hline $\begin{array}{l}\text { Composition of } \\
\text { PBT/PAr(I27-T73) }\end{array}$ & $\begin{array}{l}\text { Equilibrium melting } \\
\text { point depression of }\end{array}$ & $\begin{array}{l}\text { Equilibrium melting } \\
\text { point depression of } \\
\text { or PBT/PAr(I-100) }\end{array}$ \\
\hline PBT/PAr(I27-T73) & PBT/PAr(I-100) \\
\hline $90 / 10$ & -2.01 & -3.73 \\
$85 / 15$ & -2.37 & -6.80 \\
\hline
\end{tabular}

${ }^{\mathrm{a}}$ Equilibrium melting point depression $=T_{\mathrm{m}}^{\mathrm{o}}($ blend $)-T_{\mathrm{m}}^{\mathrm{o}}($ neat PBT $)$.

b Adopted from Ref. [10].

$220^{\circ} \mathrm{C}$, which belongs to the melting point of PBT crystallites. However, two melting endotherms (near $220^{\circ} \mathrm{C}$ and $280^{\circ} \mathrm{C}$ ) were found for the blends with compositions of $65 / 35$ and 50/50. The lower melting peak is the melting peak of PBT crystallites. The higher one is very broad and is in the range of $250{ }^{\circ} \mathrm{C}-300{ }^{\circ} \mathrm{C}$, which is the melting peak of $\operatorname{PAr}(\mathrm{I} 27-$ T73). Two distinct melting peaks show that PBT and PAr(I27-T73) crystals could coexist. Obviously, they could not form cocrystals due to different crystal structures.

The crystal structure of PAr(I27-T73) was examined by Xray diffraction. Although Fig. 1 shows that the melt-quenched PBT/PAr(I27-T73) blends of 15/85 and 0/100 did not form any crystallite, they could become semicrystalline after long-term annealing. Thus, PBT/PAr(I27-T73) 15/85 blend was quenched from $3300^{\circ} \mathrm{C}$ to $250{ }^{\circ} \mathrm{C}$ and held at $250{ }^{\circ} \mathrm{C}$ for $7 \mathrm{~h}$ to form crystallites. Results of X-ray diffraction patterns of $\operatorname{PAr}(\mathrm{I}-100)$, $\operatorname{PAr}(\mathrm{T}-100)$, and PBT/PAr(I27-T73) $15 / 85$ are shown in Fig. 6. PBT crystallites do not form in the PBT/PAr(I27-T73)

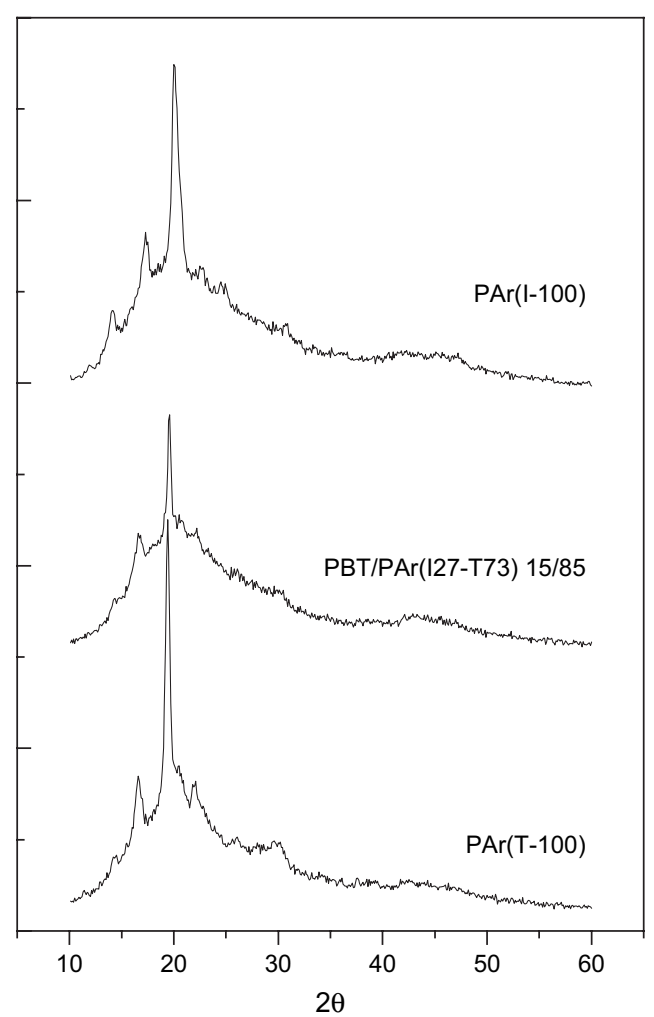

Fig. 6. X-ray diffraction patterns of isothermally crystallized PAr(I-100), PBT/ PAr(I27-T73), and PAr(T-100). 
blend in such condition. It shows that the crystal structure of PAr(I27-T73) is the same as that of PAr(T-100). Thus, crystallites of PAr(I27-T73) are composed of Bisphenol A and terephthalic, and not isophthalic segments. Also, the unit cell of PAr(I27-T73) crystal is not modified by the presence of PBT.

In Fig. 1, the recrystallization temperature of PBT increases with the increase of the amount of PAr(I27-T73). On the other hand, the crystallinity of PBT that crystallized during the quenching decreases with the increase of PAr(I27-T73) contents (see Table 1). In general, the decrease of crystallinity would be favorable to the low-temperature recrystallization due to the less constraint by crystallites, but a contrary phenomenon was observed here. Therefore, the crystallinity is not the primary effect on the recrystallization temperature. The increased recrystallization temperature is caused by the addition of high- $T_{\mathrm{g}}$ PAr(I27-T73). By adding high- $T_{\mathrm{g}}$ component, the segmental mobility is decreased and recrystallization temperature would be increased.

In addition, the DSC curve indicates that the crystallization of PBT on quenching is almost completely suppressed by PAr(I27-T73) when the weight fraction of PAr(I27-T73) is equal to or greater than $35 \%$. Furthermore, the recrystallization of PBT during the heating is suppressed by PAr(I27-T73) when the weight fraction of $\operatorname{PAr}(\mathrm{I} 27-\mathrm{T} 73)$ is equal to or greater than $65 \%$. The crystallization of PBT during the quenching and heating becomes more difficult with the increasing PAr(I27T73) contents due to the high- $T_{\mathrm{g}}$ and dilution effect of PAr(I27-T73).

Under the same process conditions, the crystallization of PBT could not be suppressed during the quenching in PBT/ PAr(I-100) blends until the weight fraction of PAr(I-100) was equal to or greater than $50 \%$ [10], and the recrystallization of PBT could not be suppressed during the heating until the PAr(I-100) composition reaches $85 \%$ [10]. Although the interactions between PBT and PAr(I27-T73) are weaker, PAr(I27T73) could suppress the crystallization of PBT more efficiently than PAr(I-100) due to the higher $T_{\mathrm{g}}$.

On the other hand, neat PAr(I27-T73) could become semicrystalline only after long-term annealing in this system. It shows that the crystallization rate of PAr(I27-T73) is very slow. Although it does not show any significant melting peak for $0 / 100,15 / 85$, and $35 / 65$, the melting peak of PAr(I27T73) was found for the blends with compositions of 50/50 and $65 / 35$ in Fig. 1. However, the crystallization of PAr(I27T73) with the composition of $85 / 15$ was suppressed again by adding more PBT. There are two major factors that influence the crystallization of PAr(I27-T73) in PBT/PAr(I27-T73) blends. PBT must be rejected out of the crystallization front during the crystallization of PAr(I27-T73), which suppresses the crystallization. Meanwhile, adding the lower $T_{\mathrm{g}}$ component, PBT, increases the segmental mobility of PAr(I27-T73), which enhances the crystallization. The crystallization of PAr(I27-T73) depends on both effects. Therefore, the crystallization of PAr(I27-T73) increases with the increase of PBT first and decreases with further addition of PBT.

The neat PAr(I-100) was also totally amorphous under the same process conditions, yet its crystallites formed as long as a relative small amount, for example, $15 \%$, of PBT was added [10]. The low- $T_{\mathrm{g}}$ effect in PBT/PAr(I-100) is more efficient than that in PBT/PAr(I27-T73). As in the blends of PBT/ PAr(I27-T73), the crystallization of PAr(I-100) was suppressed again by further addition of PBT. The crystallization of $\operatorname{PAr}(\mathrm{I}-100)$ was suppressed already when the weight ratio of PBT reached 0.65 [10], while the crystallization of PAr(I27-T73) was not suppressed at the same composition. It could be attributed to the difficulty of rejecting PBT out of crystallization front due to the greater interactions between PBT and PAr(I-100).

\subsection{Isothermal crystallization of PBT/PAr(I27-T73) blends}

The effects of PBT on the overall crystallization rate of PAr(I27-T73) are not clear in Fig. 1 due to the small crystallinity of PAr(I27-T73). Studying the isothermal crystallization kinetics of PAr(I27-T73) would be a better way to observe the PBT effects on the crystallization rate of PAr(I27-T73).

The kinetics of isothermal crystallization has been analyzed in terms of the Avrami equation [20-22] using the double logarithmic form:

$\log \left[-\ln \left(1-X_{t}\right)\right]=\log K_{n}+n \log t$

where $K_{n}$ is the overall kinetic rate constant and $n$ is the Avrami exponent related to the geometry of crystallites and the

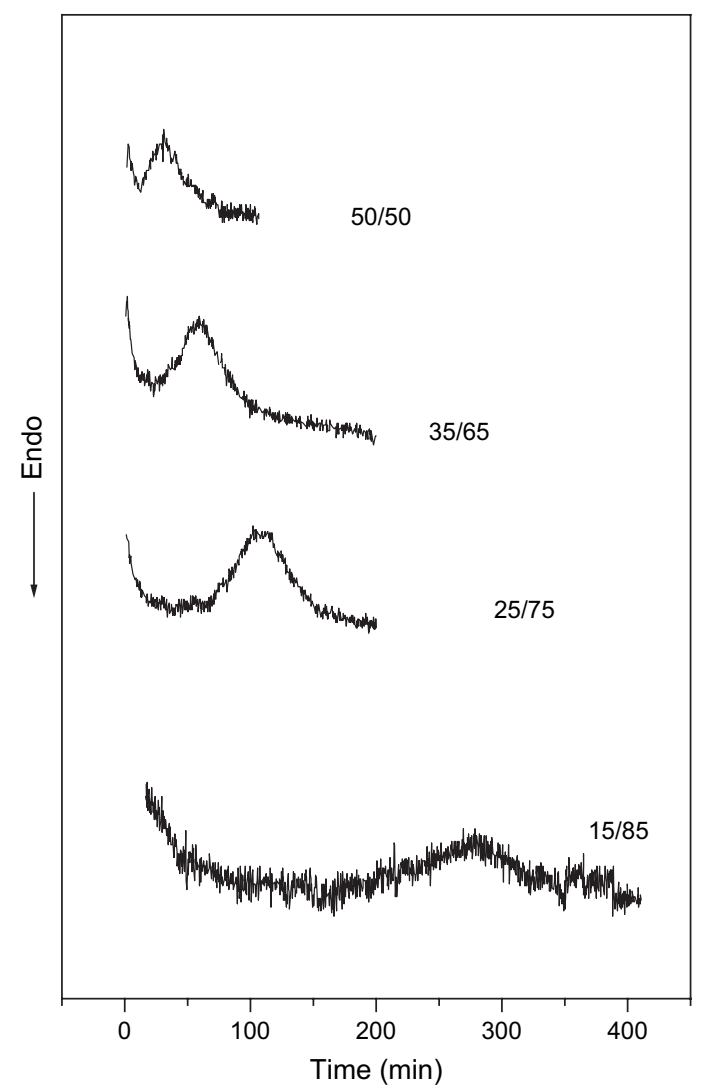

Fig. 7. The DSC traces of PBT/PAr(I27-T73) blends isothermally crystallized at $250{ }^{\circ} \mathrm{C}$. 
mechanism of the nucleation. Fig. 7 shows the DSC thermograms of PBT/PAr(I27-T73) $=50 / 50,35 / 65,25 / 75$, and 15/85 isothermally crystallized at $250{ }^{\circ} \mathrm{C}$. Only $\operatorname{PAr}(\mathrm{I} 27-\mathrm{T} 73)$ can crystallize at this temperature, and PBT cannot. The time required to reach $50 \%$ crystallization is called half-time of crystallization and is denoted as $t_{1 / 2}$. Table 4 shows the values of $t_{1 / 2}$ of the blends. The values of $t_{1 / 2}$ decrease with the increase of PBT contents. From Fig. 7 and Table 4, it was found that the crystallization rate of PAr(I27-T73) was accelerated by the increase of the amount of PBT. Although the addition of PBT, which causes depression in equilibrium melting point, would reduce the crystallization driving force, blending with PBT would lower the system $T_{\mathrm{g}}$, which increases the segmental mobility of PAr(I27-T73). Obviously, the increased segmental mobility is the major effect in this composition range.

Fig. 8 shows DSC scanning patterns of PBT/PAr(I27-T73) blends after $250{ }^{\circ} \mathrm{C}$ isothermal crystallization. The samples were isothermally crystallized at $250{ }^{\circ} \mathrm{C}$, and then were quenched from $250{ }^{\circ} \mathrm{C}$ into liquid nitrogen. The scanning rate was $20^{\circ} \mathrm{C} / \mathrm{min}$. These results are summarized in Table 5 . Although the crystallization rate of $\operatorname{PAr}($ I27-T73) is enhanced by the addition of PBT, the melting point of PAr(I27-T73)

Table 4

$t_{1 / 2}$ of PAr(I27-T73) crystallization in PBT/PAr(I27-T73) blends

\begin{tabular}{lllll}
\hline PBT/PAr(I27-T73) & $50 / 50$ & $35 / 65$ & $25 / 75$ & $15 / 85$ \\
\hline$t_{1 / 2}(\min )$ & 48.46 & 61.0 & 110.03 & 275.6 \\
\hline
\end{tabular}

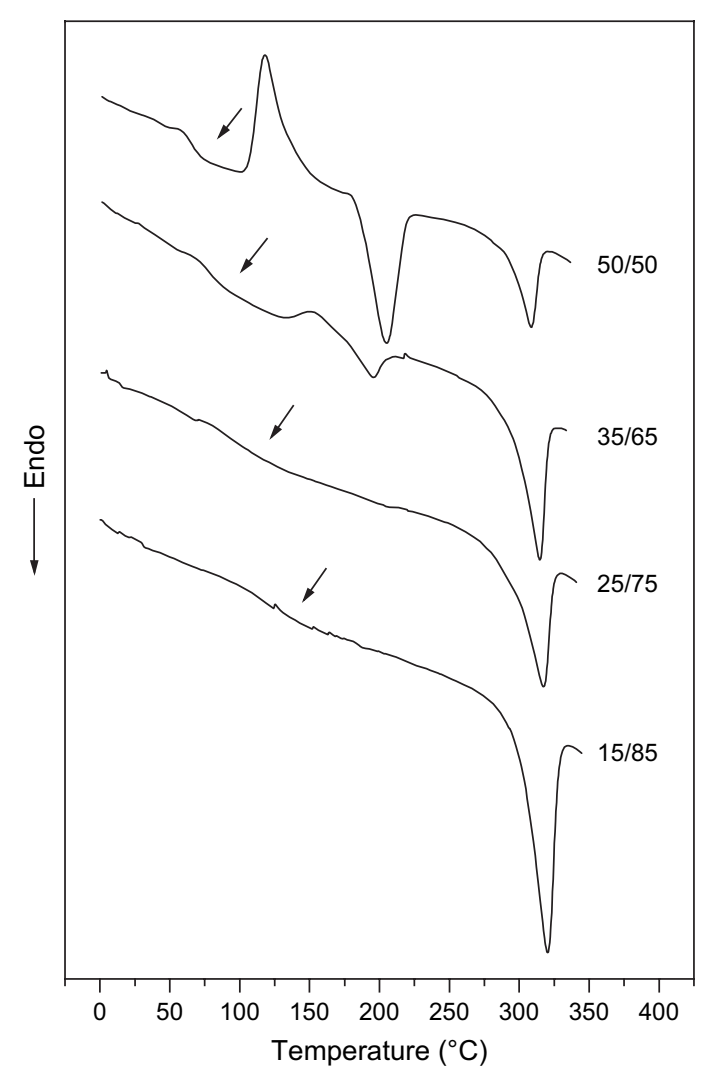

Fig. 8. The DSC traces $\left(20^{\circ} \mathrm{C} / \mathrm{min}\right)$ of PBT/PAr(I27-T73) blends after isothermal crystallized at $250^{\circ} \mathrm{C}$. and crystallinity are depressed. It can be attributed to the decrease of equilibrium melting point and the increase of defects in PAr(I27-T73) crystallites when PBT was added.

Compared with Table 1, the glass transition temperatures of all compositions are obviously lower. There are two major factors that influence the glass transition temperature. One is the previously formed PAr(I27-T73) crystallites that increase the glass transition temperature due to the constraint of crystallites. The other is the change of the composition of the amorphous phase due to the crystallization of $\operatorname{PAr}(\mathrm{I} 27-\mathrm{T} 73)$. That is, the composition of PBT in the amorphous phase is enriched, which decreases the glass transition temperature. Obviously, the latter one is dominant.

In contrast to Fig. 1, it was found in Fig. 8 that PBT would recrystallize in the scanning period in the $35 / 65$ blend after the crystallization of PAr(I27-T73). Generally, the PBT segments would be constrained by the previously formed $\operatorname{PAr}(\mathrm{I} 27-\mathrm{T} 73)$ crystallites, so it would be harder for the PBT to recrystallize. Meanwhile, the decrease of the content of PAr(I27-T73) in amorphous phase increases the mobility of PBT segment. Obviously, the change of composition of the amorphous is dominant. It should be mentioned that $T_{\mathrm{g}}$ of the blend shifts to a lower temperature $\left(78.91^{\circ} \mathrm{C}\right)$, which, calculated by Gordon-Taylor's equation, is close to $T_{\mathrm{g}}$ of a blend with the composition 54/46.

Two melting peaks of PBT/PAr(I27-T73) 50/50 are found in Fig. 8. Since the isothermal crystallization temperature is higher than the melting point of PBT, crystallites of PBT would not form during the isothermal crystallization at $250^{\circ} \mathrm{C}$. Obviously, the PBT would crystallize during the quenching and heating processes. It means that the PBT crystallites can form even in the presence of $\operatorname{PAr}($ I27-T73) crystallites. It should be mentioned that $T_{\mathrm{g}}$ of the blend shifts to a lower temperature $\left(65.15^{\circ} \mathrm{C}\right)$, which, calculated by Gordon-Taylor's equation, is close to $T_{\mathrm{g}}$ of a blend with the composition $68 / 32$. In addition, it was also found that the melting point $\left(204.95^{\circ} \mathrm{C}\right)$ and the heats of melting ( $37.28 \mathrm{~J} / \mathrm{g}$-PBT) of PBT are less than those of $65 / 35$ in Table $1\left(214.07^{\circ} \mathrm{C}\right.$ and $46.30 \mathrm{~J} / \mathrm{g}$-PBT $)$. When the $\operatorname{PAr}(\mathrm{I} 27-\mathrm{T} 73)$ crystallites previously form, they constrain the formation of PBT crystallites. Therefore, the melting point and crystallinity of PBT are depressed.

\subsection{Different heat treatments of PBT/PAr(I27-T73) blends}

Fig. 9 shows the series of DSC scans of PBT/PAr(I27$\mathrm{T} 73)=50 / 50$ blends by different heat treatments. In trace A the sample was quenched from $330{ }^{\circ} \mathrm{C}$ into liquid nitrogen (the same as in the composition 50/50 in Fig. 1). Trace B represents the blend crystallized at $150{ }^{\circ} \mathrm{C}$ for $1 \mathrm{~h}$ and then quenched to liquid nitrogen. Only PBT in the blend could crystallize during the isothermal crystallization due to the low crystallization temperature, and the blend's $T_{\mathrm{g}}$ shifted to a higher temperature $\left(105.57^{\circ} \mathrm{C}\right)$ because the content of high- $T_{\mathrm{g}}$ $\operatorname{PAr}(\mathrm{I} 27-\mathrm{T} 73)$ in the amorphous phase increased after the isothermal crystallization of PBT. The melting peaks of both PBT and PAr(I27-T73) are found in trace B, and the magnitude 
Table 5

DSC scanning data of PBT/PAr(I27-T73) blends after $250{ }^{\circ} \mathrm{C}$ isothermal crystallization

\begin{tabular}{|c|c|c|c|c|c|c|c|}
\hline \multirow[t]{2}{*}{ PBT/PAr(I27-T73) } & \multirow[t]{2}{*}{ Amorphous $T_{\mathrm{g}}\left({ }^{\circ} \mathrm{C}\right)$} & \multicolumn{3}{|c|}{ PBT phase } & \multicolumn{3}{|c|}{ PAr(I27-T73) phase } \\
\hline & & $\Delta H_{\mathrm{c}}{ }^{\mathrm{a}}$ & $\Delta H_{\mathrm{m}}$ & $T_{\mathrm{m}}\left({ }^{\circ} \mathrm{C}\right)$ & $\Delta H_{\mathrm{m}}$ & $\Delta H_{\mathrm{m}} / \mathrm{wt} \%^{\mathrm{b}}$ & $T_{\mathrm{m}}\left({ }^{\circ} \mathrm{C}\right)$ \\
\hline $50 / 50$ & 65.15 & 18.64 & 18.64 & 204.95 & 8.528 & 17.06 & 310.34 \\
\hline $35 / 65$ & 78.91 & 2.830 & 2.876 & 195.44 & 15.35 & 23.62 & 316.43 \\
\hline $25 / 75$ & 101.13 & n.a. ${ }^{\mathrm{c}}$ & n.a. & n.a. & 18.27 & 24.36 & 317.75 \\
\hline $15 / 85$ & 133.47 & n.a. & n.a. & n.a. & 25.26 & 29.72 & 319.03 \\
\hline
\end{tabular}

${ }^{a}$ Exothermic heats of recrystallization $(\mathrm{J} / \mathrm{g})$.

b Endothermic heats of melt corrected by PAr(I27-T73) weight fractions (J/g).

c Not available.

and position of the PAr(I27-T73) melting peak are comparable to those in trace $\mathrm{A}$, which implies that the formation of PAr(I27-T73) crystallites during scanning is not influenced by the previously formed PBT crystallites. In comparison, Liu et al. [10] who studied PBT/PAr(I-100) under the same conditions showed that $\operatorname{PAr}(\mathrm{I}-100)$ crystallization was completely restrained. This could be explained as follows. Trace A shows that the melting peak of PBT and recrystallization peak of PAr(I27-T73) are overlapped (see also Fig. 2). Namely, when the PBT crystal melted, the PAr(I27-T73) started to recrystallize. Thus, the formation of $\operatorname{PAr}($ I27-T73) crystallites would not be confined by PBT crystallites. In trace B, a third melting peak is found at around $160^{\circ} \mathrm{C}$, which belongs to

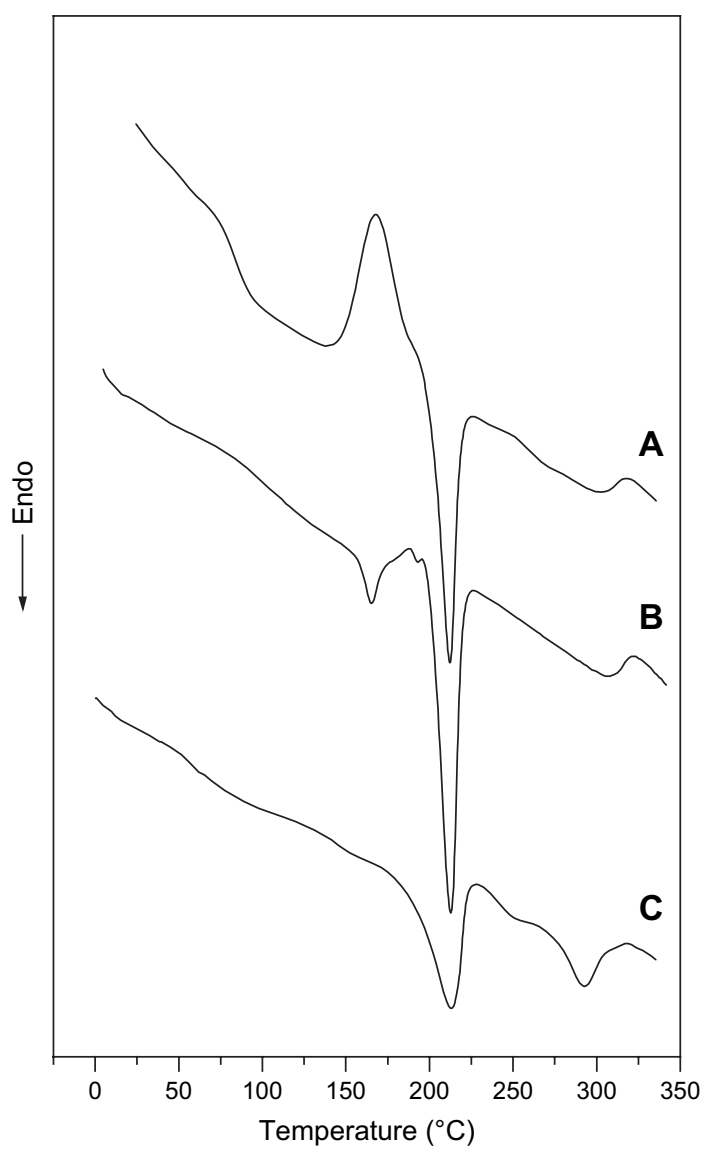

Fig. 9. The DSC traces $\left(20^{\circ} \mathrm{C} / \mathrm{min}\right)$ of PBT/PAr(I27-T73) $=50 / 50$ blend under different heat treatment conditions: (A) directly quenched from melting state, and quenched after isothermally crystallized at (B) $150{ }^{\circ} \mathrm{C}$ and (C) $200^{\circ} \mathrm{C}$.
PBT. It was reported that PBT would exhibit an extra melting point (about $10{ }^{\circ} \mathrm{C}$ higher than the isothermal crystallization temperature) after the isothermal crystallization $[23,24]$.

Trace $\mathrm{C}$ (crystallized at $200{ }^{\circ} \mathrm{C}$ for $12 \mathrm{~h}$ and then quenched to liquid nitrogen) also exhibits two melting peaks of PBT and PAr(I27-T73). Obviously, both of them can crystallize at this temperature.

\section{Conclusions}

The PBT/PAr(I27-T73) blend system shows a single composition-dependent $T_{\mathrm{g}}$ over the entire range of composition, equilibrium melting point depression of PBT phase, and a negative interaction parameter. This indicates that they are miscible in the whole range of composition.

For a miscible crystalline/crystalline blend, the glass transition temperatures and the interactions between the components will influence the crystallization behaviors. The crystallization of the component with lower glass transition temperature will be reduced by the addition of the component with higher glass transition temperature due to the decrease of chain mobility. On the other hand, the crystallization of the component with higher glass transition temperature will be increased by the addition of the component with lower glass transition temperature due to the increase of chain mobility. The weaker the interactions between components, the less the melting point depression and the easier the rejection of one component out of the crystallization front of the other.

If crystallites of one component form first, the crystallization of the other will be influenced not only by the constraint of the previously formed crystallites but also by the change of the composition of the amorphous phase.

The polyarylate used in this study, $\operatorname{PAr}(\mathrm{I} 27-\mathrm{T} 73)$, has a higher glass transition temperature and weaker interactions with PBT than PAr(I-100). In short, this affected the crystallization behavior of the blend in the following way. Because of the higher $T_{\mathrm{g}}$ of $\operatorname{PAr}(\mathrm{I} 27-\mathrm{T} 73)$, the increase of $T_{\mathrm{g}}$ of the blend was more, and so was the decrease in the segmental mobility of PBT. As a result, much less PAr(I27-T73) was needed to suppress the crystallization of PBT in the blend. In comparison, the crystallization of PAr(I27-T73) was also harder, and PBT had to be more than 50\% in the blend for PAr(I27-T73) to crystallize, while $15 \%$ PBT was enough for $\operatorname{PAr}(\mathrm{I}-100)$. However, due to the weaker interactions with PBT, PAr(I27-T73) rejected 
PBT out of the crystallization front more easily and could withstand higher concentrations of PBT until the dilution effect restrained the crystallization. Moreover, the PBT crystallites previously formed in the blend had no effect on the crystallization of PAr(I27-T73) since the melting point of PBT was lower than the crystallization temperature of the later due to the higher $T_{\mathrm{g}}$. In contrast, PAr(I-100) with lower $T_{\mathrm{g}}$ crystallized at a lower temperature and the crystallization was completely inhibited by the PBT crystallites.

\section{Acknowledgements}

We gratefully acknowledge financial support from National Science Council (NSC 89-2216-E-002-032).

\section{References}

[1] Paul DR, Barlow JW. Polymer science and technology, vol. 11. New York: Plenum Press; 1980.

[2] Runt JP, Martynowicz LM. Multicomponent polymer materials. Washington: American Chemical Society; 1986.

[3] Wu WB, Chiu WY, Liau WB. J Appl Polym Sci 1997;64:411.
[4] Domines JD. 37th ANTEC of SPE May 7-10; 1979. p. 655.

[5] Freitag D, Reinking K. Kunststoffe 1981;71(1):46.

[6] Sakata H. 32nd ANTEC of SPE April 13-16; 1974. p. 459.

[7] Runt J, Miley DM, Zhang X, Gallagher KP, McFeaters K, Fishburn J. Macromolecules 1992;25:1929.

[8] Runt J, Miley DM, Zhang X, Gallagher KP. Macromolecules 1992; 25:3902.

[9] Huo PP, Cebe P. Macromolecules 1993;26:3127.

[10] Liu AS, Liau WB, Chiu WY. Macromolecules 1998;31:6593.

[11] Liau WB, Liau AS, Chiu WY. Macromol Chem Phys 2002;203:294.

[12] Tseng TY, Chu NJ, Lee YD. J Appl Polym Sci 1990;41:1651.

[13] Devaux J, Godard P, Mercier JP. J Polym Sci Polym Phys Ed 1982; 20:1875.

[14] Kimura M, Porter RS. J Polym Sci Polym Phys Ed 1983;21:367.

[15] Illers KH. Colloid Polym Sci 1980;258:117.

[16] Fox TG. Bull Am Phys Soc 1956;1:123.

[17] Gordon M, Taylor JS. J Appl Chem 1952;2:493.

[18] Cheng SZD, Pan R, Wunderlich B. Makromol Chem 1988;189:2443.

[19] Nishi T, Wang TT. Macromolecules 1975;8:909.

[20] Avrami MJ. J Chem Phys 1939;7:1103.

[21] Avrami MJ. J Chem Phys 1940;8:212.

[22] Avrami MJ. J Chem Phys 1941;9:177.

[23] Kim J, Nichols ME, Robertson RE. J Polym Sci Polym Phys 1994;32:887

[24] Kim HG, Robertson RE. J Polym Sci Polym Phys 1998;36:1757. 